\section{Fatores associados à adequação do cuidado pré-natal e à assistência ao parto em São Tomé e Príncipe, 2008-2009}

\author{
Factors associated with adequate prenatal care \\ and delivery in São Tomé and Príncipe, 2008-2009 \\ Factores asociados con la adecuación del cuidado \\ prenatal y de la asistencia al parto en Santo Tomé \\ y Príncipe, 2008-2009
}

Patrícia Alexandra da Graça Dantas dos Reis 1 Claudia Cristina de Aguiar Pereira 2 Iuri da Costa Leite 2 Mariza Miranda Theme-Filha ${ }^{2}$

\section{Resumo}

Neste artigo, foram identificados fatores sociodemográficos associados com o cuidado prénatal e com a assistência ao parto em São Tomé e Príncipe. A amostra foi composta por 1.326 nascidos vivos de mulheres de 15 a 49 anos que participaram do Inquérito Demográfico e Sanitário de São Tomé e Príncipe, 2008-2009. Foram utilizados modelos de regressão logística e multinomial multiníveis. A adequação global do cuidado pré-natal foi de $26 \%$ e da assistência ao parto de 7\% quando realizado por médicos e de 76\% quando realizado por enfermeiras/ auxiliares. Os fatores associados ao pré-natal e à assistência ao parto adequados foram: ordem de nascimento, educação materna e o índice de bem-estar econômico. O local de residência se mostrou fator importante apenas em relação à assistência ao parto. Observou-se que os efeitos aleatórios referentes às áreas onde as mulheres residem exerceram impacto importante sobre a chance de realizar pré-natal adequado e parto com profissionais capacitados. A importância dos fatores socioeconômicos aponta para a elaboração de ações que visem reduzir a desigualdade social em São Tomé e Príncipe.

Parto; Cuidado Pré-Natal; Tocologia 


\section{Introdução}

Aprimorar a atenção à saúde materna é um dos oito objetivos adotados pela Cúpula do Milênio em 2000. Estabeleceu-se como principal meta reduzir, em $75 \%$, a razão de mortalidade materna (RMM) entre 1990 e 2015. Estimativas da Organização das Nações Unidas (ONU), para o ano de 2005 , mostram que a razão de mortalidade materna declinou a uma taxa de $2,3 \%$ ao ano, valor bem abaixo da taxa de 5,5\% ao ano, necessária para que a meta do milênio seja atingida em todo o mundo ${ }^{1 .}$

A morte materna, definida como o óbito de uma mulher durante a gravidez ou no período de 42 dias após a gestação, permanece como um grande desafio a ser enfrentado pelos serviços de saúde, principalmente nos países em desenvolvimento, onde há fortes evidências de falhas nos serviços de atenção primária 2,3. O acesso aos serviços de saúde adequados é apontado como um meio eficaz de reduzir os riscos de mortalidade e morbidade materna, principalmente entre as populações mais vulneráveis 4 .

Os benefícios do cuidado pré-natal sobre a saúde da mãe e da criança têm sido destacados em vários estudos 5,6,7,8. O cuidado pré-natal possibilita a detecção precoce de problemas de saúde, permitindo, assim, o tratamento oportuno, de modo a evitar complicações durante a gravidez e no parto 4,8,9,10,11,12. A assistência ao parto também desempenha um papel relevante na redução da mortalidade e da morbidade materna. Ele deve ser realizado em ambiente adequado e com a presença de um profissional capacitado para assegurar que qualquer tipo de complicação seja devidamente controlada 13,14,15.

Um dos grandes desafios para os países em desenvolvimento é dispor de atenção à saúde de forma equânime, oportuna e efetiva. Em vista da importância do cuidado pré-natal na prevenção de mortes maternas e perinatais, a Organização Mundial da Saúde (OMS) preconiza a realização de um conjunto mínimo de procedimentos e intervenções, fundamentados em evidências científicas, dirigidos a todas as gestantes e realizados em momentos críticos da gravidez. Tais procedimentos são passíveis de serem aplicados em qualquer contexto social e de organização da atenção 1,16,17,18.

A despeito das vantagens do cuidado pré-natal, sua qualidade é ainda insuficiente nos países em desenvolvimento, não logrando alcançar os efeitos desejados sobre a saúde materno-infantil. Entretanto, o termo "adequação do cuidado" nem sempre é definido de forma uniforme. Pode incluir o número de visitas 19,20,21, início do prénatal 22,23 ou continuidade do provedor do cui- dado 12,13. Além disso, os indicadores que mensuram as trajetórias da definição da adequação do pré-natal e da assistência ao parto têm sido distintos, produzindo uma variedade de critérios de adequação que conduzem a diferentes interpretações de resultados 24 .

Alguns autores 6,17 sugerem que índices mais abrangentes sejam criados, uma vez que o refinamento desses resultaria, em princípio, numa melhoria das ferramentas para monitorizar o cuidado recebido pela mulher e melhor avaliação em conformidade com os padrões estabelecidos, melhorando, assim, as atuais políticas de saúde e programas de intervenção 19,20.

Tendo em vista a elevada RMM na África Subsaariana e a importância dos indicadores de saúde no processo de redução da mortalidade materna, estudos têm procurado identificar fatores sociodemográficos associados à utilização do cuidado pré-natal e à assistência ao parto, utilizando informações de países dessa região $14,15,16,17,18,22,23$. Até o momento, nenhum estudo sobre esse tema foi conduzido em São Tomé e Príncipe, país de língua portuguesa, com população em torno de 163 mil habitantes e predominantemente jovem. Em 2008, a RMM de São Tomé e Príncipe foi estimada em 150 mortes por 100 mil nascidos vivos 24 , valor relativamente baixo quando comparado ao da África Subsaariana, mas bastante elevado quando observado junto aos números dos países desenvolvidos, que se encontram em torno de sete mortes maternas por 100 mil nascidos vivos 24 .

Nesse contexto, o governo de São Tomé e Príncipe lançou, na década de 2000, o Plano Nacional de Desenvolvimento Sanitário. Entre outras metas, o plano inclui a melhoria do acesso aos serviços de saúde. Recentemente, foi realizada uma pesquisa de âmbito nacional, a qual permitiu a obtenção de um conjunto de informações detalhadas sobre a saúde das mulheres em idade reprodutiva, incluindo dados referentes aos cuidados pré-natais e à assistência ao parto 25 .

Não se tem conhecimento de artigos ou publicações que abordassem a qualidade da assistência ao pré-natal e ao parto em São Tomé e Príncipe. Dessa forma, o objetivo deste estudo é investigar a associação entre fatores socioeconômicos e demográficos e a assistência ao pré-natal e ao parto em São Tomé e Príncipe, utilizando um estudo de base populacional.

\section{Métodos}

No presente estudo utilizamos as recomendações mínimas propostas pela OMS 26,27 para definir a adequabilidade do pré-natal e expandi- 
mos o conceito de adequabilidade da assistência ao parto por meio da desagregação do tipo de profissional que realizou o parto. Os dados analisados foram obtidos do Inquérito Demográfico e Sanitário, realizado em São Tomé e Príncipe, entre setembro de 2008 e março de 2009 (IDS-STP-2008-09), pelo Instituto Nacional de Estatística (INE) e pelo Ministério da Saúde 25. A pesquisa faz parte do programa Demographic and Health Survey (DHS) cujo objetivo principal é prover informações nacionalmente representativas para o monitoramento e a avaliação de um conjunto de indicadores das áreas de população e saúde nos países em desenvolvimento. Tratase de uma amostra probabilística em dois estágios: no primeiro estágio foram selecionados os setores censitários; no segundo, os domicílios, nos quais todas as mulheres em idade reprodutiva foram entrevistadas. A amostra foi composta de 2.615 mulheres de 15 a 49 anos e de 1.931 nascidos vivos nos últimos cinco anos. Informações sobre o cuidado pré-natal foram dadas apenas para a última gravidez, compreendendo 1.445 nascidos vivos. Foram excluídas observações nas quais não tinham sido preenchidas as informações sobre o mês de início do pré-natal nem sobre o profissional que realizou o parto. Assim, a análise se desenvolveu com base em informações sobre 1.326 nascimentos, o equivalente a uma perda de $8,2 \%$.

Dois desfechos foram analisados: adequação do cuidado pré-natal e da assistência ao parto por profissional qualificado. A variável desfecho, referente à adequabilidade do pré-natal, foi adaptada a partir das recomendações da OMS, que prevê a realização de quatro consultas prénatais, sendo a primeira entre 8 e 12 semanas gestacionais; realização de exames de sangue para dosagem de hemoglobina, identificação do grupo sanguíneo e fator Rh, testagem para sífilis e HIV; realização de exame de urina para pesquisa de bacteriúria e proteinúria; imunização com toxoide tetânico; tratamento intermitente preventivo da malária; prescrição de folatos e de sulfato ferroso; avaliação clínica e obstétrica, incluindo a aferição da pressão arterial em todas as consultas e avaliação do crescimento fetal e dos batimentos cardiofetais a partir da segunda consulta 26,27 .

Com base nas informações disponíveis no IDS-STP-2008-09 25, considerou-se como pré-natal adequado aquele que cumprisse todos os critérios a seguir: início nos primeiros três meses de gravidez; realização de, pelo menos, quatro consultas; pelo menos, uma mensuração do peso e da altura da mãe; pelo menos, uma mensuração da altura uterina e da pressão arterial; realização de exame de sangue para dosagem de hemoglo- bina e teste de VDRL e HIV; realização de exame de urina; prescrição do sulfato ferroso e ausculta dos batimentos cardiofetais.

A ausência das informações necessárias quanto ao número de doses da vacina antitetânica e a profilaxia da malária impossibilitaram o uso dessas intervenções na construção do indicador de adequabilidade.

Em relação à assistência ao trabalho de parto, a OMS recomenda que seja realizada por profissional de saúde capacitado com formação e treinamento para o manejo de gravidezes normais (não complicadas), sendo capaz de identificar e dar resolutividade aos casos complicados 27 .

$\mathrm{Na}$ impossibilidade de aferir, com base nas informações disponíveis, se o parto foi realizado por profissional capacitado, considerou-se a assistência ao parto adequada quando foi realizada por médico, enfermeiro ou auxiliar de enfermagem e inadequada quando foi realizada por parteiras tradicionais, familiares ou amigos 28,29. Dessa forma, três categorias nominais para quem realizou o parto foram criadas. Duas categorias para profissionais de saúde considerados como capacitados: médico e enfermeira/parteira/parteira auxiliar e uma para outras pessoas: parteira tradicional/amigas/familiares/outra.

Há fortes evidências científicas de que indivíduos selecionados de uma mesma área geográfica são mais propensos a apresentar comportamento e atitudes mais semelhantes do que indivíduos selecionados de áreas diferentes, caracterizando assim uma estrutura hierárquica nos dados e, consequentemente, correlação entre as observações de uma mesma área. Modelos de regressão tradicionais assumem independência entre as observações e, quando aplicados a dados com estrutura hierárquica, tendem a produzir, de forma espúria, parâmetros estatisticamente significativos. Para contornar esse problema, modelos multiníveis foram utilizados 30 , tratando o setor censitário como um efeito aleatório.

$\mathrm{Na}$ análise da adequabilidade do pré-natal, por termos apenas duas categorias (adequado, inadequado), utilizou-se um modelo logístico multinível, com dois níveis: o setor censitário onde a mãe reside e o nível do nascido vivo/mãe. Em relação à análise da adequabilidade da assistência ao parto, pelo fato de a variável desfecho possuir três categorias, um modelo multinomial multinível foi utilizado com três categorias nominais acerca de quem realizou o parto.

O processo de estimação e seleção das variáveis se deu em duas etapas. Inicialmente, realizou-se a análise bivariada cujas variáveis significativas no nível de $25 \%$ foram selecionadas para o modelo multivariado. No modelo final, permaneceram apenas as variáveis estatisticamente 
significativas no nível de significância de 5\%. Os parâmetros dos modelos multiníveis foram estimados utilizando-se o software MlwiN (Centre for Multilevel Modelling, Bristol, Reino Unido), com base no procedimento de quase verossimilhança preditiva (PQL) de segunda ordem, que, segundo Goldstein \& Rasbash 31 , produzem estimativas mais fidedignas.

Um conjunto de variáveis sociodemográficas, apontadas na literatura como associadas com os desfechos de interesse e disponíveis na base de dados na pesquisa IDS-STP-2008-09 25, foi incorporado às análises desenvolvidas.

Todos os dados utilizados neste estudo foram obtidos por meio de fonte de domínio público, sem identificação dos participantes, dispensando, portanto, a apreciação pelo sistema de comitês de ética (CEP/CONEP).

\section{Resultados}

No que se refere à adequação do cuidado prénatal, o primeiro critério a ser analisado foi o início da primeira consulta pré-natal até o 3o mês de gestação. Para esse item, verificou-se que mais de um terço das mulheres (44\%) iniciou o pré-natal no primeiro trimestre. Apesar de se constatar um início tardio do cuidado prénatal (56\%), se comparado ao número total de consultas durante a gestação, constatou-se que aproximadamente oito em cada dez mulheres (78\%) fizeram, pelo menos, as quatro consultas recomendadas. Neste estudo, a realização dos procedimentos de medição da pressão arterial, peso, altura uterina e dos batimentos cardiofetais foi elevada, próxima dos $100 \%$, semelhante à observada em outros locais 32,33,34,35. Além disso, nota-se que $95 \%$ das mulheres receberam complementos de ferro (comprimido ou xarope). A medição da altura da gestante apresentou proporções mais baixas (74\%), o que sugere a necessidade de uma maior inclusão à rotina do pré-natal. A realização de exames de hemoglobina atingiu $87,4 \%$, os de urina, HIV e VDRL alcançaram, respectivamente, 89,7\%, 86\% e 85,8\%. Assim, a adequação global do cuidado pré-natal foi de $25,7 \%$.

Na Tabela 1 são apresentadas as distribuições das características da população sob investigação e as proporções de ocorrência de cada desfecho analisado segundo essas características. Sobre os aspectos individuais, trata-se de uma população com idade entre 20 e 34 anos de idade $(67 \%)$, casadas $(87 \%)$, com três filhos ou mais (62\%), tendo desejado engravidar do último filho (50\%), com educação primária (74\%) e religião predominantemente católica (70\%).
Conforme o esperado, o índice de bem-estar econômico 25 apresenta uma distribuição bem equânime, em que a alocação percentual de cada renda dos domicílios girava em torno de $20 \%$. Por fim, quase $60 \%$ das mulheres vivem em áreas rurais.

$\mathrm{Na}$ Tabela 2 são apresentadas as razões de chance e os respectivos intervalos de confiança dos modelos de regressão logística multinível, não ajustados para a adequação do cuidado prénatal. Sobre os fatores individuais, observou-se que quanto maior a idade da mulher, menor a chance de efetuar um cuidado pré-natal adequado, ou seja, as mulheres com menos de 20 anos tiveram $82 \%$ de chances de terem um cuidado adequado quando comparadas às mulheres com 35 anos ou mais ( $\mathrm{RC}=1,82$; IC95\%: 1,13-2,95). Mães com menos filhos (1-2) registraram uma chance $107 \%$ maior de adequação ao pré-natal quando comparadas às que possuíam três filhos ou mais ( $R C=2,07$; IC95\%: 1,56-2,74). A variável educação revelou que quanto maior o nível educacional, maior a chance de cuidado adequado. Mulheres com nível secundário/superior tiveram aproximadamente cinco vezes mais chances do que as que não possuem qualquer nível educacional (RC = 5,94; IC95\%: 2,43-14,52).

Os fatores domiciliares mostraram uma relação direta entre o nível de bem-estar econômico e a chance de adequação do cuidado pré-natal. Assim, a chance de realizar o pré-natal adequado entre mulheres pertencentes ao quintil de renda mais elevada foi $160 \%$ maior do que a chance observada entre mulheres pertencentes ao quintil de renda mais baixo ( $\mathrm{RC}=2,57$; IC95\%: 1,59-4,16).

No modelo ajustado, as variáveis estatisticamente significativas avaliadas ao nível de 5\% foram: ordem de nascimento, nível educacional e índice de bem-estar econômico (Tabela 3). As variáveis mostraram o mesmo sentido observado no modelo não ajustado. Entretanto, observouse uma diminuição dos efeitos sobre o desfecho. A variável ordem de nascimento perdeu cerca de 10\% do seu efeito, o nível educacional secundário/superior perdeu, aproximadamente, $33 \%$, e a categoria de bem-estar econômico mais elevado (muito elevado) teve seu efeito reduzido em $23 \%$.

Na Tabela 4 são exibidas as razões de chance e os intervalos de confiança do modelo multinomial multinível não ajustado para o desfecho assistência ao parto, nos dois níveis avaliados como adequados (médico e enfermeira/auxiliar). A variável ordem de nascimento mostrou que mulheres com menos filhos tiveram maiores chances de adequação na assistência, tanto em relação à participação de um médico $(\mathrm{RC}=$ 3,32; IC95\%: 1,65-6,70) quanto de enfermeira/ auxiliar (RC = 2,28; IC95\%: 1,61-3,24). A variável 
Distribuição das mulheres por tipo de pré-natal e profissional que realizou o parto segundo um conjunto de características selecionadas.

\begin{tabular}{|c|c|c|c|c|c|c|c|c|c|c|}
\hline \multirow[t]{3}{*}{ Características } & \multirow[t]{3}{*}{$\mathrm{n}$} & \multirow[t]{3}{*}{$\%$} & \multirow{2}{*}{\multicolumn{2}{|c|}{$\begin{array}{c}\text { Cuidado pré-natal } \\
\text { Adequado }\end{array}$}} & \multicolumn{6}{|c|}{ Assistência ao parto } \\
\hline & & & & & \multicolumn{2}{|c|}{ Médico } & \multicolumn{2}{|c|}{ Enfermeira/Auxiliar } & \multicolumn{2}{|c|}{ Outros } \\
\hline & & & $\mathrm{n}$ & $\%$ & $\mathrm{n}$ & $\%$ & $\mathrm{n}$ & $\%$ & $\mathrm{n}$ & $\%$ \\
\hline \multicolumn{11}{|l|}{ Fatores individuais } \\
\hline \multicolumn{11}{|l|}{ Idade materna (anos) } \\
\hline$\leq 19$ & 189 & 14,25 & 57 & 30,16 & 14 & 7,41 & 155 & 82,01 & 20 & 10,58 \\
\hline $20-34$ & 885 & 66,74 & 229 & 25,88 & 55 & 6,21 & 657 & 74,24 & 173 & 19,55 \\
\hline 35 ou mais & 252 & 19,00 & 55 & 21,83 & 17 & 6,75 & 189 & 75,00 & 46 & 18,25 \\
\hline \multicolumn{11}{|l|}{ Ordem de nascimento } \\
\hline $1-2$ & 501 & 37,78 & 167 & 33,33 & 37 & 7,39 & 407 & 81,24 & 57 & 11,38 \\
\hline 3 ou mais & 825 & 62,22 & 174 & 21,09 & 49 & 5,94 & 594 & 72,00 & 182 & 18,02 \\
\hline \multicolumn{11}{|l|}{ Estado civil } \\
\hline Casada/Unida & 1.154 & 87,03 & 52 & 30,23 & 76 & 6,59 & 870 & 75,39 & 208 & 18,02 \\
\hline Outros & 172 & 12,97 & 289 & 25,04 & 10 & 5,81 & 131 & 76,76 & 31 & 18,02 \\
\hline \multicolumn{11}{|l|}{ Religião } \\
\hline Católica & 924 & 69,68 & 251 & 22,39 & 27 & 6,72 & 304 & 75,62 & 71 & 17,66 \\
\hline Outras & 402 & 30,32 & 90 & 27,16 & 59 & 6,39 & 697 & 75,43 & 168 & 18,18 \\
\hline \multicolumn{11}{|l|}{ Educação } \\
\hline Nenhuma & 70 & 5,28 & 7 & 10,00 & 2 & 2,86 & 54 & 77,14 & 14 & 20,00 \\
\hline Primária & 980 & 73,91 & 222 & 22,65 & 53 & 5,41 & 725 & 73,98 & 202 & 20,61 \\
\hline Secundária/Superior & 276 & 20,81 & 112 & 40,58 & 31 & 11,23 & 222 & 80,43 & 23 & 8,33 \\
\hline \multicolumn{11}{|l|}{ Desejava engravidar } \\
\hline Naquele momento & 668 & 50,38 & 178 & 26,65 & 37 & 5,54 & 516 & 77,25 & 115 & 17,22 \\
\hline Mais tarde & 371 & 27,98 & 105 & 28,30 & 31 & 8,36 & 272 & 73,32 & 68 & 18,33 \\
\hline Não queria mais filhos & 287 & 21,64 & 58 & 20,21 & 18 & 6,27 & 213 & 74,22 & 56 & 19,51 \\
\hline \multicolumn{11}{|l|}{ Fatores domiciliares } \\
\hline \multicolumn{11}{|c|}{ Índice de bem-estar econômico } \\
\hline Muito baixo & 304 & 22,93 & 61 & 20,07 & 11 & 3,62 & 219 & 72,04 & 74 & 24,34 \\
\hline Baixo & 311 & 23,45 & 68 & 21,86 & 20 & 6,43 & 215 & 69,13 & 76 & 24,44 \\
\hline Médio & 286 & 21,57 & 66 & 23,08 & 18 & 6,29 & 219 & 76,57 & 49 & 17,13 \\
\hline Elevado & 253 & 19,08 & 73 & 28,85 & 17 & 6,72 & 206 & 81,42 & 30 & 11,86 \\
\hline Muito elevado & 172 & 12,97 & 73 & 42,44 & 20 & 11,63 & 142 & 82,56 & 10 & 5,81 \\
\hline \multicolumn{11}{|l|}{ Fatores da comunidade } \\
\hline \multicolumn{11}{|l|}{ Local de residência } \\
\hline Rural & 788 & 59,43 & 169 & 21,45 & 38 & 4,82 & 570 & 72,34 & 180 & 22,84 \\
\hline Urbano & 538 & 40,67 & 172 & 31,97 & 48 & 8,92 & 431 & 80,11 & 59 & 10,97 \\
\hline Total & 1.326 & 100,00 & 341 & 25,72 & 86 & 6,49 & 1.001 & 75,49 & 239 & 18,02 \\
\hline
\end{tabular}

Fonte: Inquérito Demográfico e Sanitário, São Tomé e Príncipe, 2008-2009 (IDS-STP-2008-09) 25.

educação mostrou que a adequação na assistência por parte do médico foi tão maior quanto o nível educacional, sendo que as mulheres que tinham educação secundária/superior apresentaram uma chance bem mais elevada do que as mulheres sem nenhuma educação ( $R C=9,90$; IC95\%: 1,41-69,49). Com relação à categoria enfermeira/auxiliar, a escolaridade apresentou valores mais baixos ( $\mathrm{RC}=2,33$; IC95\%: 1,06-5,09). Nos fatores domiciliares, observou-se uma curva ascendente, ou seja, quanto mais elevado o bem- estar econômico, maior a adequação, independentemente da categoria profissional analisada. Mulheres do quintil mais elevado de bem-estar econômico registraram chances muito maiores de serem assistidas de maneira adequada por um médico do que mulheres do quintil mais baixo (RC = 15,03; IC95\%: 4,12-54,80). Já a adequação na assistência por parte das enfermeiras/auxiliares apresenta valores mais modestos, fazendo-se a mesma comparação (RC = 4,54; IC95\%: 2,139,69). As mulheres do meio urbano pontuaram 
Tabela 2

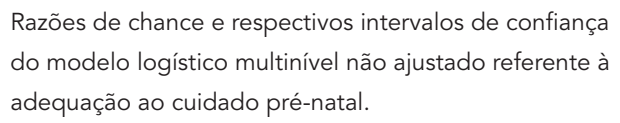

\begin{tabular}{|c|c|c|}
\hline Características & $\mathrm{RC}$ & IC95\% \\
\hline \multicolumn{3}{|l|}{ Fatores individuais } \\
\hline \multicolumn{3}{|l|}{ Idade materna (anos) } \\
\hline$\leq 19$ & 1,82 & $1,13-2,95$ \\
\hline $20-34$ & 1,40 & $0,96-2,03$ \\
\hline 35 ou mais & 1,00 & \\
\hline \multicolumn{3}{|l|}{ Ordem de nascimento } \\
\hline $1-2$ & 2,07 & $1,56-2,74$ \\
\hline 3 ou mais & 1,00 & \\
\hline \multicolumn{3}{|l|}{ Estado civil } \\
\hline Casada/Unida & 0,66 & $0,44-0,98$ \\
\hline Outros & 1,00 & \\
\hline \multicolumn{3}{|l|}{ Religião } \\
\hline Católica & 1,32 & $0,96-1,80$ \\
\hline Outras & 1,00 & \\
\hline \multicolumn{3}{|l|}{ Educação } \\
\hline Nenhuma & 1,00 & \\
\hline Primária & 2,82 & $1,19-6,67$ \\
\hline Secundária/Superior & 5,94 & $2,43-14,52$ \\
\hline \multicolumn{3}{|l|}{ Desejava engravidar } \\
\hline Naquele momento & 1,00 & \\
\hline Mais tarde & 0,96 & $0,70-1,32$ \\
\hline Não queria mais filhos & 0,59 & $0,40-0,86$ \\
\hline \multicolumn{3}{|l|}{ Fatores domiciliares } \\
\hline \multicolumn{3}{|c|}{ Índice de bem-estar econômico } \\
\hline Muito baixo & 1,00 & \\
\hline Baixo & 1,29 & $0,85-1,98$ \\
\hline Médio & 1,28 & $0,83-1,99$ \\
\hline Elevado & 1,88 & $1,20-2,92$ \\
\hline Muito elevado & 2,57 & $1,59-4,16$ \\
\hline \multicolumn{3}{|l|}{ Fatores da comunidade } \\
\hline \multicolumn{3}{|l|}{ Local de residência } \\
\hline Rural & 1,00 & \\
\hline Urbano & 1,69 & $1,03-2,76$ \\
\hline
\end{tabular}

IC95\%: intervalo de 95\% de confiança; RC: razão de chances.

Fonte: Inquérito Demográfico e Sanitário, São Tomé e Príncipe, 2008-2009 (IDS-STP-2008-09) 25.

maior chance de realizar a assistência com um médico ( $\mathrm{RC}=6,16$; IC95\%: 2,49-15,26) e enfermeira/auxiliar (RC = 2,44; IC95\%: 1,56-3,83) do que as mulheres residentes no meio rural.

No modelo ajustado, apenas as variáveis ordem de nascimento, índice de bem-estar econômico e o local de residência foram estatisticamente relevantes ao nível de 5\% (Tabela 5).
Tabela 3

Razões de chance e respectivos intervalos de confiança do modelo logístico multinível ajustado referente à adequação ao cuidado pré-natal.

\begin{tabular}{|c|c|c|}
\hline Características & $\mathrm{RC}$ & IC95\% \\
\hline \multicolumn{3}{|l|}{ Fatores individuais } \\
\hline \multicolumn{3}{|l|}{ Ordem de nascimento } \\
\hline $1-2$ & 1,87 & $1,40-2,50$ \\
\hline 3 ou mais & 1,00 & \\
\hline \multicolumn{3}{|l|}{ Educação } \\
\hline Nenhuma & 1,00 & \\
\hline Primária & 2,56 & $1,07-6,13$ \\
\hline Secundária/Superior & 4,01 & $1,59-10,09$ \\
\hline \multicolumn{3}{|l|}{ Fatores domiciliares } \\
\hline \multicolumn{3}{|c|}{ Índice de bem-estar econômico } \\
\hline Muito baixo & 1,00 & \\
\hline Baixo & 1,27 & $0,83-1,96$ \\
\hline Médio & 1,24 & $0,79-1,93$ \\
\hline Elevado & 1,61 & $1,02-2,55$ \\
\hline Muito elevado & 1,99 & $1,19-3,34$ \\
\hline \multicolumn{3}{|c|}{$\begin{array}{l}\text { IC95\%: intervalo de 95\% de confiança; RC: razão de } \\
\text { chances. }\end{array}$} \\
\hline \multicolumn{3}{|c|}{ Fonte: Inquérito Demográfico e Sanitário, São Tomé e } \\
\hline \multicolumn{3}{|c|}{ Príncipe, 2008-2009 (IDS-STP-2008-09) 25.} \\
\hline
\end{tabular}

Apesar de não ter sido significativa, a variável nível educacional foi mantida no modelo, pois apresentou um efeito muito forte em relação à assistência ao parto ter sido feita por um médico. Na realidade, o nível educacional não se mostrou estatisticamente significativo, pois apresentou um erro-padrão bastante alto.

Em relação à ordem de nascimento, observou-se que a chance de as mulheres com um ou dois filhos terem uma assistência ao parto realizada por médico e enfermeira/auxiliar foi, respectivamente, cerca de quatro a duas vezes maiores do que a observada entre mulheres com três ou mais filhos. O efeito do nível educacional correspondente ao secundário/superior teve sua intensidade reduzida, mas a chance de ter um parto realizado por um médico continuou elevada (RC = 3,90; IC95\%: 0,48-31,57).

As mulheres com nível de bem-estar econômico mais elevado apresentaram chance de ter um parto com médico quase dez vezes maior àquela observada entre mulheres do nível mais baixo de bem-estar econômico, o que significa um decréscimo de aproximadamente $37 \%$. A variável local de residência também teve seu efeito reduzido, embora com menos intensidade. $\mathrm{Mu}$ lheres residentes em áreas urbanas foram mais 
Tabela 4

Razões de chance e respectivos intervalos de confiança do modelo multinomial multinível não ajustado referente à adequação da assistência ao parto.

\begin{tabular}{|c|c|c|c|c|}
\hline \multirow[t]{2}{*}{ Características } & \multicolumn{2}{|c|}{ Médico } & \multicolumn{2}{|c|}{ Enfermeira/Auxiliar } \\
\hline & $\mathrm{RC}$ & IC95\% & $\mathrm{RC}$ & IC95\% \\
\hline \multicolumn{5}{|l|}{ Fatores individuais } \\
\hline \multicolumn{5}{|l|}{ Idade materna (anos) } \\
\hline$\leq 19$ & 3,13 & $0,98-10,02$ & 2,05 & $1,12-3,76$ \\
\hline $20-34$ & 0,95 & $0,40-2,25$ & 0,92 & $0,63-1,36$ \\
\hline 35 ou mais & 1,00 & & 1,00 & \\
\hline \multicolumn{5}{|l|}{ Ordem de nascimento } \\
\hline $1-2$ & 3,32 & $1,65-6,70$ & 2,28 & $1,61-3,24$ \\
\hline 3 ou mais & 1,00 & & 1,00 & \\
\hline \multicolumn{5}{|l|}{ Estado civil } \\
\hline Casada/Unida & 0,83 & $0,31-2,12$ & 0,94 & $0,60-1,48$ \\
\hline Outros & 1,00 & & 1,00 & \\
\hline \multicolumn{5}{|l|}{ Religião } \\
\hline Católica & 1,03 & $0,51-2,07$ & 1,01 & $0,72-1,41$ \\
\hline Outras & 1,00 & & 1,00 & \\
\hline \multicolumn{5}{|l|}{ Educação } \\
\hline Nenhuma & 1,00 & & 1,00 & \\
\hline Primária & 1,64 & $0,26-10,18$ & 0,90 & $0,47-1,72$ \\
\hline Secundária/Superior & 9,90 & $1,41-69,49$ & 2,33 & $1,06-5,09$ \\
\hline \multicolumn{5}{|l|}{ Desejava engravidar } \\
\hline Naquele momento & 1,00 & & 1,00 & \\
\hline Mais tarde & 1,58 & $0,73-3,40$ & 0,85 & $0,59-1,23$ \\
\hline Não queria mais filhos & 1,08 & $0,45-2,59$ & 0,76 & $0,51-1,13$ \\
\hline \multicolumn{5}{|l|}{ Fatores domiciliares } \\
\hline \multicolumn{5}{|c|}{ Índice de bem-estar econômico } \\
\hline Muito baixo & 1,00 & & 1,00 & \\
\hline Baixo & 2,22 & $0,83-5,98$ & 1,02 & $0,69-1,51$ \\
\hline Médio & 2,77 & $0,97-7,93$ & 1,62 & $1,05-2,52$ \\
\hline Elevado & 5,18 & $1,69-15,88$ & 2,28 & $1,35-3,76$ \\
\hline Muito elevado & 15,03 & $4,12-54,80$ & 4,54 & $2,13-9,69$ \\
\hline \multicolumn{5}{|l|}{ Fatores da comunidade } \\
\hline \multicolumn{5}{|l|}{ Local de residência } \\
\hline Rural & 1,00 & & 1,00 & \\
\hline Urbano & 6,16 & $2,49-15,26$ & 2,44 & $1,56-3,83$ \\
\hline
\end{tabular}

IC95\%: intervalo de 95\% de confiança; RC: razão de chances.

Fonte: Inquérito Demográfico e Sanitário, São Tomé e Príncipe, 2008-2009 (IDS-STP-2008-09) 25.

propensas a ter filho com médicos $(\mathrm{RC}=5,32$; IC95\%: 2,15-3,18) e enfermeiras/auxiliares ( $\mathrm{RC}=$ 1,98; IC95\%: 1,26-3,11) quando comparadas às mulheres do meio rural.

A magnitude dos efeitos aleatórios indica que existe uma considerável parcela da variabilidade entre as áreas onde as mães residem não explicada pelas variáveis incluídas no modelo. Assim, devem existir outros fatores relativos à mãe ou à área de residência das mães, mensu- ráveis ou não, que possivelmente expliquem essa variabilidade. Os coeficientes de correlação intraclasse - que expressam o quanto da variação não explicada é relacionado às áreas nas quais as mulheres residem - mostraram-se elevados: $23,59 \%$ no cuidado pré-natal adequado e $34,41 \%$ e $12,06 \%$ na assistência ao parto por médico e enfermeira/auxiliar, respectivamente (Tabela 6). 
Razões de chance e respectivos intervalos de confiança do modelo multinomial multinível ajustado referente à adequação da assistência ao parto.

\begin{tabular}{|c|c|c|c|c|}
\hline \multirow[t]{2}{*}{ Características } & \multicolumn{2}{|c|}{ Médico } & \multicolumn{2}{|c|}{ Enfermeira/Auxiliar } \\
\hline & $\mathrm{RC}$ & IC95\% & $\mathrm{RC}$ & IC95\% \\
\hline \multicolumn{5}{|l|}{ Fatores individuais } \\
\hline \multicolumn{5}{|l|}{ Ordem de nascimento } \\
\hline $1-2$ & 3,68 & $1,75-7,74$ & 2,13 & $1,50-3,03$ \\
\hline 3 ou mais & 1,00 & & 1,00 & \\
\hline \multicolumn{5}{|l|}{ Educação } \\
\hline Nenhuma & 1,00 & & 1,00 & \\
\hline Primária & 1,49 & $0,22-10,01$ & 0,79 & $0,41-1,52$ \\
\hline Secundária/Superior & 3,90 & $0,48-31,57$ & 1,19 & $0,53-31,57$ \\
\hline \multicolumn{5}{|l|}{ Fatores domiciliares } \\
\hline \multicolumn{5}{|c|}{ Índice de bem-estar econômico } \\
\hline Muito baixo & 1,00 & & 1,00 & \\
\hline Baixo & 2,29 & $0,80-6,54$ & 0,98 & $0,66-1,46$ \\
\hline Médio & 2,79 & $0,92-8,46$ & 1,56 & $0,77-3,16$ \\
\hline Elevado & 3,94 & $1,18-13,12$ & 2,10 & $1,26-3,51$ \\
\hline Muito elevado & 9,50 & $2,24-40,35$ & 3,30 & $1,50-7,24$ \\
\hline \multicolumn{5}{|l|}{ Fatores da comunidade } \\
\hline \multicolumn{5}{|l|}{ Local de residência } \\
\hline Rural & 1,00 & & 1,00 & \\
\hline Urbano & 5,32 & $2,15-13,18$ & 1,98 & $1,26-3,11$ \\
\hline
\end{tabular}

IC95\%: intervalo de 95\% de confiança; RC: razão de chances.

Fonte: Inquérito Demográfico e Sanitário, São Tomé e Príncipe, 2008-2009 (IDS-STP-2008-09) 25.

Tabela 6

Efeitos aleatórios e os coeficientes de correlação intraclasse referentes aos modelos multiníveis logístico do cuidado pré-natal e multinomial da assistência ao parto.

\begin{tabular}{|c|c|c|c|}
\hline \multirow[t]{2}{*}{ Parâmetros } & \multirow{2}{*}{$\begin{array}{c}\text { Cuidado pré-natal } \\
\text { adequado }\end{array}$} & \multicolumn{2}{|c|}{ Assistência ao parto adequada } \\
\hline & & Médico & Enfermeira/Auxiliar \\
\hline \multicolumn{4}{|c|}{ Efeito aleatório } \\
\hline$\sigma^{2} u$ & 1,02 * & 1,73 * & 0,45 * \\
\hline \multicolumn{4}{|c|}{ Coeficiente de correlação intraclasse } \\
\hline$\rho$ & $23,59 \%$ & $34,41 \%$ & $12,06 \%$ \\
\hline
\end{tabular}

* $p<0,05$.

Fonte: Inquérito Demográfico e Sanitário, São Tomé e Príncipe, 2008-2009 (IDS-STP-2008-09) 25.

O efeito da área de residência pode então exercer um impacto expressivo sobre os desfechos. Para ilustrar esse fato, na Tabela 7, são apresentadas as probabilidades de uma mulher realizar o cuidado pré-natal adequado e ser assistida durante o parto por um médico, segundo a educação materna e níveis de efeitos aleatórios referentes às áreas onde as mulheres residem (2 desvios-padrão abaixo da média, 1 desvio padrão abaixo da média, média, 1 desvio padrão acima da média e 2 desvios padrão acima da média). Essas probabilidades foram calculadas tomando-se todas as demais variáveis do modelo no valor médio 36,37. Como pode ser observado, 
Probabilidades de realizar o cuidado pré-natal adequado e o parto com médico, segundo a educação materna e níveis de efeito aleatório referente à área de residência: dois desvios-padrão abaixo da média, um desvio padrão abaixo da média, média, um desvio padrão acima da média, dois desvios-padrão acima da média.

\begin{tabular}{|c|c|c|c|c|c|}
\hline \multirow[t]{2}{*}{ Tipo de cuidado/Nível educacional } & \multicolumn{5}{|c|}{ Nível do efeito aleatório } \\
\hline & -2 & -1 & 0 & 1 & 2 \\
\hline \multicolumn{6}{|l|}{ Cuidado pré-natal adequado } \\
\hline \multicolumn{6}{|l|}{ Educação } \\
\hline Nenhuma & 0,019 & 0,048 & 0,118 & 0,262 & 0,484 \\
\hline Primária & 0,045 & 0,110 & 0,247 & 0,465 & 0,696 \\
\hline Secundária/Superior & 0,070 & 0,165 & 0,344 & 0,344 & 0,786 \\
\hline \multicolumn{6}{|l|}{ Parto realizado por médico } \\
\hline \multicolumn{6}{|l|}{ Educação } \\
\hline Nenhuma & 0,002 & 0,007 & 0,118 & 0,086 & 0,260 \\
\hline Primária & 0,003 & 0,110 & 0,044 & 0,147 & 0,391 \\
\hline Secundária/Superior & 0,006 & 0,165 & 0,079 & 0,241 & 0,541 \\
\hline
\end{tabular}

Fonte: Inquérito Demográfico e Sanitário, São Tomé e Príncipe, 2008-2009 (IDS-STP-2008-09) 25.

os efeitos aleatórios referentes às áreas de residência das mães podem neutralizar os ganhos obtidos por meio do aumento dos anos de escolaridade. Assim, tanto na adequação do cuidado pré-natal como na da assistência ao parto, mulheres sem nenhuma educação, residentes em áreas situadas a dois desvios-padrão acima da média apresentam probabilidade de realizar cuidado pré-natal e assistência ao parto de forma adequada maior do que as que apresentam educação secundária/superior e são residentes em áreas situadas a dois desvios-padrão abaixo da média.

\section{Discussão}

O objetivo deste trabalho foi investigar a associação entre fatores socioeconômicos e demográficos e a adequação da assistência pré-natal e do parto em São Tomé e Príncipe. Este artigo teve por base o primeiro Inquérito Demográfico e Sanitário conduzido em São Tomé e Príncipe. Os resultados encontrados, por meio da análise empregada, podem ser considerados únicos em termos de conteúdo, cobertura geográfica em São Tomé e Príncipe, possibilitando, assim, um conhecimento mais detalhado do estado da saúde materna nesse país.

O estudo contribui para uma ampliação do conceito de adequação do cuidado pré-natal uma vez que a definição de pré-natal adequado foi mais ampla do que aquela na qual se considera apenas o mês de início e o número de consul- tas realizadas. Da mesma forma, o conceito de adequabilidade da assistência ao parto também foi expandido na medida em que se diferenciaram as duas categorias consideradas como adequadas: médico e enfermeira/auxiliar.

A adequação global do cuidado pré-natal foi de $25,7 \%$, bem aquém daquela obtida quando levamos, em consideração, o número mínimo de quatro consultas ou a realização da primeira consulta nos primeiros três meses de gestação 1,26,27, com, respectivamente, $44 \%$ e $78,4 \%$ das mulheres.

As variáveis que se mostraram estatisticamente significativas em relação à adequação do cuidado pré-natal foram a ordem de nascimento, o nível educacional materno e o índice de bemestar econômico. Em relação à assistência adequada ao parto, além dessas variáveis, a variável referente ao local de residência também se mostrou estatisticamente significativa.

O resultado observado para a variável ordem de nascimento corrobora outros dados nos quais mulheres cujos filhos são de ordem ao nascer baixa apresentam maior propensão a receber o pré-natal e assistência ao parto adequadas. Resultados semelhantes foram encontrados em estudos sobre a Turquia e a Índia 38,39. Segundo esses autores, mulheres de maior parturição tendem a acreditar que as experiências de partos anteriores se repetirão e, portanto, não consideram tão necessário o acompanhamento do pré-natal durante a gestação ${ }^{38,39}$. Isso poderia explicar o fato de as mulheres mais jovens apresentarem maior chance de fazer pré- 
natal com profissional capacitado no modelo não ajustado.

Outras três variáveis não foram estatisticamente significativas tanto em relação ao prénatal quanto à assistência ao parto adequados. O estado civil mostrou-se associado com o prénatal adequado apenas no modelo não ajustado com menor chance de ser realizado entre as mulheres casadas/unidas, contrapondo achados da literatura ${ }^{40}$. A ausência de associação entre o desejo de engravidar e os desfechos analisados vai de encontro a resultados de estudos que destacam que mulheres que não desejavam engravidar naquele momento ou não queriam ter mais filhos apresentam menores taxas de utilização dos serviços de saúde 9. Embora haja evidências de que a religião possa influenciar a utilização dos serviços de saúde, principalmente por meio da hipótese de seletividade, na qual um grupo religioso poderia ter maior acesso ao capital social, que, por sua vez, aumentaria seu acesso aos serviços de saúde. Neste estudo, nenhuma relação foi observada 41 .

O índice de bem-estar econômico apareceu como um forte indicativo da atenção pré-natal e da assistência ao parto adequadas, conforme observado em outros estudos 9,40. Mulheres com maiores níveis de bem-estar econômico tiveram mais chances de terem um cuidado pré-natal mais satisfatório e de contarem com um profissional na assistência ao parto do que as mulheres com menor nível de bem-estar econômico.

Observou-se ainda que as mulheres com maior nível educacional e local de residência urbano tiveram maior oportunidade de um cuidado pré-natal e assistência ao parto adequados, em consonância com os resultados observados em outros estudos 29,38,40,42. Mulheres com maior nível educacional teriam mais informações sobre os benefícios do cuidado pré-natal e dos exames necessários para o bem-estar da criança. Além disso, há fortes evidências de que mulheres mais expostas à educação formal são mais conducentes a utilizar os serviços obstétricos 9 . Já o efeito do local de residência atuaria como uma proxy do acesso aos serviços de saúde, geralmente distribuídos de forma desproporcional entre os locais de residência, favorecendo os centros urbanos, principalmente em relação à alocação de médicos e enfermeiras/auxiliares 9 .
O início do cuidado pré-natal tem como grande vantagem a detecção precoce de eventuais complicações durante a gestação, como hipertensão arterial crônica, diabetes não gestacional, anemia, anemia pela sífilis e pelo HIV. Tais problemas, em muitos casos, podem ser evitados, prevenidos ou tratados quando a gestante e o bebê têm acesso a métodos de diagnóstico e tratamento eficazes. Países como os Estados Unidos e a Inglaterra preconizam o início do pré-natal até as 12 primeiras semanas de gestação 43,44 período também considerado como adequado no Brasil 45.

Algumas limitações sobre esse estudo merecem ser destacadas: a impossibilidade de incorporar todos os critérios propostos pela OMS para a adequação do pré-natal devido à limitação da DHS; a falta de informações sobre a disponibilidade/acessibilidade dos serviços, o que limita a utilização prática dos resultados, principalmente nas áreas rurais onde os fatores ligados à localização, aos recursos humanos e aos insumos são preponderantes no uso dos serviços maternos e o possível viés de memória devido ao fato de o estudo utilizar dados obtidos em entrevista materna relativa à última gestação ocorrida nos cinco anos anteriores à data da entrevista. No que diz respeito às perdas de informações, é possível que essas tenham sido seletivas, ou seja, tenham ocorrido entre mulheres com menor nível de escolaridade e residentes nas áreas rurais, tornando as estimativas para essas variáveis mais conservadoras.

Mesmo diante de algumas simplificações efetuadas por falta de dados disponíveis sobre a temporalização dos diversos procedimentos como, por exemplo, não considerar o momento na gravidez em que os exames foram realizados consideramos que as análises realizadas constituem uma importante contribuição para a literatura sobre saúde materna e infantil em São Tomé e Príncipe, podendo ser estendida a outros países africanos.

Espera-se que os achados desse estudo sejam utilizados na elaboração de recomendações para ações, programas ou políticas de saúde visando a uma maior adequação do cuidado pré-natal e a melhor assistência ao parto. 


\section{Resumen}

En este artículo se identificaron factores demográficos $y$ socioeconómicos, asociados al cuidado prenatal, y a la asistencia al parto en Santo Tomé y Príncipe. La muestra estaba compuesta 1.326 recién nacidos de mujeres de 15 a 49 años, procedentes del Informe Demográfico y Sanitario de Santo Tomé y Príncipe, 2008-2009, integrado en la Demographic and Health Survey. Se utilizaron modelos de regresión logística y multinomial multiniveles. En la adecuación, el cuidado prenatal fue de un $26 \%$ y la asistencia al parto fue de un $7 \%$ por parte de médicos y de un $76 \%$ por parte de enfermeras/auxiliares. Los factores significativamente asociados al cuidado prenatal fueron: el orden del nacimiento, el nivel educacional materno y el índice de bienestar económico. Entre los factores más importantes de asistencia al parto fueron: orden de nacimiento, nivel educacional, índice de bienestar económico y lugar de residencia. Los factores socioeconómicos jugaron un rol relevante sobre el cuidado adecuado del período prenatal y la asistencia al parto, señalando acciones para reducir la desigualdad social en Santo Tomé y Príncipe.

Parto; Atención Prenatal; Tocología

\section{Colaboradores}

P. A. G. D. Reis foi responsável pela concepção e desenho do estudo, executou a análise dos dados, participou da elaboração e revisão do manuscrito e aprovou a versão final. C. C. A. Pereira e I. C. Leite colaboraram no desenho do estudo, análise dos dados, elaboração e revisão do manuscrito e aprovaram a versão final. M. M. Theme-Filha contribuiu no desenho do estudo, interpretação dos dados e revisão crítica do conteúdo, participou da elaboração do texto e aprovou a versão final.

\section{Agradecimentos}

A autora P. A. G. D. Reis agradece ao CNPq pela bolsa de doutorado que possibilitou a realização do trabalho.

\section{Referências}

1. World Health Organization; United Nations Children's Fund; United Nations Population Fund; World Bank. Trends in maternal mortality 19902008: estimates developed by WHO, UNICEF, UNFPA and The World Bank. Geneva:World Health Organization; 2010.

2. Obaid TA. Fifteen years after the International Conference on Population and Development: what have we achieved and how we move forward? Int J Gynaecol Obstet 2009; 106:102-5.

3. Hogan MC, Foreman KJ, Naghavi M, Ahn S, Wang M, Makela SM, et al. Maternal mortality for 181 countries, 1980-2008: a systematic analysis of progress towards Millennium Development Goal 5. Lancet 2010; 375:1609-23.

4. Reynolds H, Wong E, Tucker H. Adolescents use of maternal and child health services in developing countries. Int Fam Plan Perspect 2006; 32:6-16.

5. Hueston WJ, Gilbert GE, Davis L, Sturgill V. Delayed prenatal care and the risk of low birth weight delivery. J Community Health 2003; 28:199-208.

6. Viellas EF, Domingues RMSM, Dias MAB, Gama SGN, Theme Filha MM, Costa JV, et al. Assistência pré-natal no Brasil. Cad Saúde Pública 2014; 30 Suppl:S85-100.

7. Corrêa MD, Tsunechiro NA, Lima MOP, Bonadio IS. Avaliação da assistência pré-natal em unidade com estratégia saúde da família. Rev Esc Enferm USP 2014; 48:24-32.
8. Nwaru BI, Wu Z, Hemminki E. Determinants of the use of prenatal care in rural China: the role of care content. Matern Child Health J 2012; 16:235-41.

9. Magadi MA, Madise NJ, Rodrigues RN. Frequency and timing of antenatal care in Kenya: explaining the variations between women of different communities. Soc Sci Med 2000; 51:551-61.

10. Magadi MA, Zulu EM, Borckerhoff M. The inequality of maternal health care inurban sub-Saharan Africa in the 1990s. Popul Stud (Camb) 2003; 57:347-66.

11. Adam T, Lim S, Mehta S, Bhutta ZA, Fogstad H, Mathai $M$, et al. Cost effectiveness analysis of strategies for maternal and neonatal health in developing countries. BMJ 2005; 331:1-6.

12. McCaw-Binns A, Lagrenade J, Ashley D. Underusers of antenatal care: a comparison of non-attenders and late attenders for antenatal care with early attenders. Soc Sci Med 2007; 40:1003-12.

13. Villar J, Ba'aqeel H, Piaggio G, Lumbiganon P, Belizán J, Farnot U, et al. WHO antenatal care randomised trial for the evaluation of new model of routine antenatal care. Lancet 2001; 357:1551-64.

14. Ochako R, Fotso JC, Ikamari L, Khasakhala A. Utilization of maternal health services among young women in Kenya: insights from the Kenya Demographic and Health Survey, 2003. BMC Pregnancy Childbirth 2011; 11:1-9. 
15. Magadi MA, Agwanda AO, Obare FA. A comparative analysis of the use of maternal health services between teenagers and older mothers in sub-Saharan Africa: evidence from Demographic and Health Surveys (DHS). Soc Sci Med 2007; 64:1311-25.

16. Kestler E. Wanted: better care for pregnant women. World Health Forum 1993; 14:356-9.

17. Munjanja SP, Lindmark G, Nyström L. Randomised controlled trial of a reduced-visits programme ofantenatal care in Harare, Zimbabwe. Lancet 1996; 348:364-9.

18. Villar J, Khan-Neelofur D. Patterns of routine carefor low-risk pregnancy. Patterns of routine antenatalcare for low-risk pregnancy. Cochrane Database Syst Rev 2001; 4:CD000934.

19. Raatikainen K, Heiskanen N, Heinonen S. Underattending free antenatal care is associated with adverse outcomes. BMC Public Health 2007; 7:1-8.

20. Walker DS, McCully L, Vest V. Evidence-based prenatal care visits: when less is more. J Midwifery Womens Health 2001; 46:146-51.

21. Petrou S, Kupek E, Vause S, Maresh M. Clinical, provider and sociodemographic determinants of the number of antenatal visits in England and Wales. Soc Sci Med 2001; 52:1123-34.

22. Nothnagle M, Marchi K, Egerter S, Braveman P. Risk factors for late or no prenatal care following Medicaid expansions in California. Matern Child Health J 2000; 4:251-9.

23. Reichman NE, Kenney GM. Prenatal care, birth outcomes and newborn hospitalization costs: patterns among Hispanics in New Jersey. Fam Plann Perspect 1998; 30:182-200.

24. Barros AJD, Ronsmans C, Axelson H, Bertoldi AD, França GVA, Bryce J, et al. Equity in maternal, newborn, and child health interventions in Countdown to 2015: a retrospective review of survey data from 54 countries. Lancet 2012; 379:1225-33.

25. Instituto Nacional de Estatística; Ministério da Saúde; ICF Macro. Inquérito demográfico e sanitário, São Tomé e Príncipe (IDS STP, 2008-2009). Calverton: Instituto Nacional de Estatística; 2010.

26. World Health Organization. Pregnancy, childbirth, postpartum and newborn care: a guide for essential practice. Geneva: World Health Organization; 2003.

27. Department of Making Pregnancy Safer, World Health Organization. Integrated management of pregnancy and childbirth: WHO recommended interventions for improving maternal and newborn health. Geneva: World Health Organization; 2009.

28. Crissman HP, Engmann CE, Adanu RM, Nimako D, Crespo K, Moyer CA. Shifting norms: pregnant women's perspectives on skilled birth attendance and facility-based delivery in rural Ghana. Afr J Reprod Health 2013; 17:15-26.

29. Vallières F, Hansen A, McAuliffe E, Cassidy EL, Owora P, Kappler S, et al. Head of household education level as a factor influencing whether delivery takes place in the presence of a skilled birth attendant in Busia, Uganda: a cross-sectional household study. BMC Pregnancy Childbirth 2013; 13:48.

30. Gelman A, Hill J. Data analysis using regression and multilevel/hierarchical models. New York: Cambridge University Press; 2007.
31. Goldstein H, Rasbash J. Improved approximations for multilevel models with binary responses. J R Stat Soc Ser A Stat Soc 1996; 159:505-13.

32. Domingues RMSM, Hartz ZMA, Dias MAB, Leal MC. Avaliação da adequação da assistência prénatal na rede SUS do Município do Rio de Janeiro, Brasil. Cad Saúde Pública 2012; 28:425-37.

33. Ribeiro ERO, Gimarães AMDN, Bettiol H, Lima DDF, Almedida ML, Souza L, et al. Risk factors for inadequate prenatal care use in the metropolitan area of Aracaju, Northeast Brazil. BMC Pregnancy Childbirth 2009; 9:1-8.

34. Coutinho T, Monteiro MFG, Sayd JD, Teixeira MTB, Coutinho CM, Coutinho LM. Monitoring the prenatal care process among users of the Unified Health Care System in a city of the Brazilian Southeast. Rev Bras Ginecol Obstet 2010; 32:563-9.

35. Andreucci CB, Cecatti JG. Desempenho de indicadores de processo do Programa de Humanização do Pré-natal e Nascimento no Brasil: uma revisão sistemática. Cad Saúde Pública 2011; 27:1053-64.

36. Curtis SL, Diamond I, McDonald JW. Family effects on postneonatal mortality in Brazil. Demography 1993; 30:33-44.

37. Pebley AR, Goldman N, Rodríguez G. Prenatal and delivery care and childhood immunization in Guatemala: do family and community matter? Demography 1996; 33:231-47.

38. Celik Y, Hotchkiss DR. The socio-economic determinants of maternal health care utilization in Turkey. Soc Sci Med 2000; 50:1797-806.

39. Pallikadavath S, Foss M, Stones RW. Antenatal care: provision and inequality in rural north India. Soc Sci Med 2004; 59:1147-58.

40. Simkhada B, van Teijlingen ER, Porter M, Simkhada P. Factors affecting the utilization of antenatal care in developing countries: systematic review of the literature. J Adv Nurs 2008; 61:244-60

41. Ha W, Salama P, Gwavuya S, Kanjala C. Is religion the forgotten variable in maternal and child health? Evidence from Zimbabwe. Soc Sci Med 2014; 118:80-8.

42. Furuta M, Salway S. Women's position within the household as a determinant of maternal care use in Nepal. Int Fam Plan Perspect 2006; 32:17-27.

43. National Institute for Health and Clinical Excellence. Antenatal care: NICE Clinical Guideline 62. London: National Institute for Health and Clinical Excellence; 2010

44. American College of Obstetricians and Gynecologists. Standards for obstetric-gynecologic services. Washington DC: American College of Obstetricians and Gynecologists; 1985.

46. Ministério da Saúde. Portaria consolidada Rede Cegonha. http://bvsms.saude.gov.br/bvs/saudele gis/gm/2011/prt1459_24_06_2011.html (acessado em 22/Dez/2014).

Recebido em 05/Ago/2014

Versão final reapresentada em 21/Jan/2015 Aprovado em 05/Fev/2015 